\title{
ON SIMPLE 15-DIMENSIONAL LIE ALGEBRAS IN CHARACTERISTIC 2
}

\author{
ALEXANDER GRISHKOV, HENRIQUE GUZZO JR., MARINA RASSKAZOVA, AND PASHA ZUSMANOVICH
}

\begin{abstract}
Motivated by the recent progress towards classification of simple finite-dimensional Lie algebras over an algebraically closed field of characteristic 2, we investigate such 15-dimensional algebras.
\end{abstract}

\section{INTRODUCTION}

The classification of finite-dimensional simple Lie algebras over an algebraically closed field has a long and interesting history. The characteristic zero case, due to Killing, Cartan, and Dynkin, is nowadays a classic. The case of characteristic $p>3$ was accomplished relatively recently, due to the efforts of dozens of people, spread over more than 50 years and hundreds of papers, and culminated in the three volumes of Strade, [St]. The cases of characteristics 2 and 3 remain open, although a lot of efforts were done recently to augment the classification program by small characteristics specifics, in particular, to put Lie superalgebras into play (see, for example, a monumental treatise by Leites and his collaborators, [BGLLS], and references therein).

In [GZ], we did another small step towards the classification of finite-dimensional simple Lie algebras over an algebraically closed field of characteristic 2 : it was proved that any such algebra of absolute toral tank 2, and having a Cartan subalgebra of toral rank one, is 3-dimensional. In this, we based on the paper of Skryabin, [Sk], where it was proved, among other things, that in characteristic 2 there are no simple Lie algebras of absolute toral rank 1, and simple Lie algebras having a Cartan subalgebra of toral rank 1 where characterized as certain filtered deformations of semisimple Lie algebras with the socle of the form $S \otimes \mathcal{O}$, where $S$ is a simple Lie algebra either of Zassenhaus or Hamiltonian type, and $\mathcal{O}$ is a reduced polynomial algebra.

In the process of the proof of the main result of [GZ], we have constructed a 2-parameter family $\mathscr{L}(\beta, \delta)$ of 15 -dimensional simple Lie algebras $([\mathrm{GZ}, \S 6])$. The algebra $\mathscr{L}(0,0)$ in this family coincides with the smallest algebra in the series constructed by Skryabin ([Sk, Example at pp. 691-692]).

It is the purpose of this paper to study the family $\mathscr{L}(\beta, \delta)$. Among other things, we prove that all algebras within the family are isomorphic to the same algebra $\mathscr{L}(\S 1)$, and we determine the absolute toral rank (\$3), and the automorphism group of $\mathscr{L}(\$ 4)$. In passing, we introduce the notion of a thin decomposition of a simple Lie algebra with respect to a torus (\$3.4) which, we suggest, should play a role in classification efforts (see open questions in \$7).

Throughout the paper, the ground field $K$ is assumed to be perfect of characteristic 2 , unless stated otherwise. Although the initial problem assumes the ground field is algebraically closed, for our purposes it will be enough to assume that square roots exist in $K$. This allows to include in our consideration the case $K=\mathrm{GF}(2)$, what can be useful in some circumstances (cf. $\$ 3$ and $\S 6$ ).

Our terminology and notation is mostly standard. $C_{L}(X)$ denotes the centralizer of a set $X$ in a Lie algebra $L$; the linear span of a set $X$ is denoted either as $K X$ or as $\langle X\rangle$; the 2-envelope of a Lie algebra $L$ is denoted by $L_{2} ; \mathrm{id}_{L}$ denotes the identity map on $L$. Other notation is explained as soon as it is introduced in the text. 


\section{The 2-PARAMETER FAMily $\mathscr{L}(\beta, \delta)$}

Recall the definition of the family $\mathscr{L}(\beta, \delta)$, depending of two parameters $\beta, \delta \in K$, of 15-dimensional simple Lie algebras constructed in [GZ, §6]. These algebras are filtered deformations of the semisimple Lie algebra of the form

$$
\mathrm{S} \otimes \mathcal{O}_{1}(2)+g \otimes\langle 1, x\rangle+\partial,
$$

where $\mathrm{S}$ is the 3 -dimensional simple Lie algebra with the basis $\{e, f, h\}$ and multiplication table

$$
[e, h]=e, \quad[f, h]=f, \quad[e, f]=h,
$$

$g=(\operatorname{ad} f)^{2}$ is an outer derivation of $\mathrm{S}, \mathcal{O}_{1}(2)$ is the 4-dimensional divided power algebra with the basis $\left\{1, x, x^{(2)}, x^{(3)}\right\}$, and $\partial: x^{(i)} \rightarrow x^{(i-1)}$ is the special derivation of $\mathcal{O}_{1}(2)$.

For our purpose, it will be convenient to relabel the basis elements of the algebras $\mathscr{L}(\beta, \delta)$ as follows:

$$
\begin{array}{ll}
b_{1}=e \otimes 1 & c_{1}=e \otimes x^{(3)} \\
b_{2}=f \otimes 1 & c_{2}=f \otimes x^{(3)} \\
b_{3}=h \otimes 1 & c_{3}=h \otimes x^{(3)} \\
b_{4}=e \otimes x & c_{4}=g \otimes 1 \\
b_{5}=f \otimes x & c_{5}=g \otimes x \\
b_{6}=h \otimes x & d=\partial \\
b_{7}=e \otimes x^{(2)} & \\
b_{8}=f \otimes x^{(2)} & \\
b_{9}=h \otimes x^{(2)} &
\end{array}
$$

In terms of this basis, the multiplication table of $\mathscr{L}(\beta, \delta)$ (see [GZ, (5.4)]) reads:

(3)

\begin{tabular}{|c||c|c|c|c|c|c|c|c|c|c|c|c|c|c|}
\hline & $b_{2}$ & $b_{3}$ & $b_{4}$ & $b_{5}$ & $b_{6}$ & $b_{7}$ & $b_{8}$ & $b_{9}$ & $c_{1}$ & $c_{2}$ & $c_{3}$ & $c_{4}$ & $c_{5}$ & $d$ \\
\hline \hline$b_{1}$ & $b_{3}$ & $b_{1}$ & $\beta c_{5}$ & $b_{6}$ & $b_{4}$ & $\delta c_{4}$ & $b_{9}$ & $b_{7}$ & $\delta c_{5}+d$ & $c_{3}$ & $c_{1}$ & $b_{2}$ & $b_{5}$ & $\beta c_{2}$ \\
\hline$b_{2}$ & & $b_{2}$ & $b_{6}$ & 0 & $b_{5}$ & $b_{9}$ & 0 & $b_{8}$ & $c_{3}$ & 0 & $c_{2}$ & 0 & 0 & 0 \\
\hline$b_{3}$ & & & $b_{4}$ & $b_{5}$ & 0 & $b_{7}$ & $b_{8}$ & 0 & $c_{1}$ & $c_{2}$ & 0 & 0 & 0 & 0 \\
\hline$b_{4}$ & & & & 0 & 0 & $\delta c_{5}+d$ & $c_{3}$ & $c_{1}$ & $b_{3}$ & $c_{4}$ & $b_{2}$ & $b_{5}$ & 0 & $b_{1}$ \\
\hline$b_{5}$ & & & & & 0 & $c_{3}$ & 0 & $c_{2}$ & $c_{4}$ & 0 & 0 & 0 & 0 & $b_{2}$ \\
\hline$b_{6}$ & & & & & & $c_{1}$ & $c_{2}$ & 0 & 0 & 0 & $c_{4}$ & 0 & 0 & $b_{3}$ \\
\hline$b_{7}$ & & & & & & & 0 & 0 & $b_{6}$ & $c_{5}$ & $b_{5}$ & $b_{8}$ & $c_{2}$ & $b_{4}$ \\
\hline$b_{8}$ & & & & & & & & 0 & $c_{5}$ & 0 & 0 & 0 & 0 & $b_{5}$ \\
\hline$b_{9}$ & & & & & & & & & 0 & 0 & $c_{5}$ & 0 & 0 & $b_{6}$ \\
\hline$c_{1}$ & & & & & & & & & & 0 & $b_{8}$ & $c_{2}$ & 0 & $b_{7}$ \\
\hline$c_{2}$ & & & & & & & & & & & 0 & 0 & 0 & $b_{8}$ \\
\hline$c_{3}$ & & & & & & & & & & & & 0 & 0 & $b_{9}$ \\
\hline$c_{4}$ & & & & & & & & & & & & & 0 & 0 \\
\hline$c_{5}$ & & & & & & & & & & & & & & $c_{4}$ \\
\hline
\end{tabular}

Lemma 1. For any $\beta, \delta \in K$, we have $\mathscr{L}(\beta, \delta) \simeq \mathscr{L}(\beta, 0)$.

Proof. Let us consider a new basis

$$
\left\{b_{1}^{\prime}, b_{2}, b_{3}^{\prime}, b_{4}^{\prime}, b_{5}, b_{6}, b_{7}^{\prime}, b_{8}, b_{9}^{\prime}, c_{1}^{\prime}, c_{2}, c_{3}, c_{4}, c_{5}, d^{\prime}\right\}
$$


of the algebra $\mathscr{L}(\beta, \delta)$, where

$$
\begin{aligned}
& b_{1}^{\prime}=b_{1}+\sqrt{\delta} b_{6}+\delta b_{8} \\
& b_{3}^{\prime}=b_{3}+\sqrt{\delta} b_{5} \\
& b_{4}^{\prime}=b_{4}+\delta c_{2} \\
& b_{7}^{\prime}=b_{7}+\sqrt{\delta} c_{3} \\
& b_{9}^{\prime}=b_{9}+\sqrt{\delta} c_{2} \\
& c_{1}^{\prime}=c_{1}+\sqrt{\delta} c_{4} \\
& d^{\prime}=d+\sqrt{\delta} b_{2} .
\end{aligned}
$$

It is straightforward to check that in this basis the multiplication table coincides with the multiplication table (3) with $\delta=0$.

Lemma 2. For any $\beta \in K$, we have $\mathscr{L}(\beta, 0) \simeq \mathscr{L}(0,0)$.

Proof. Consider a new basis

$$
\left\{b_{1}^{\prime}, b_{2}, b_{3}^{\prime}, b_{4}^{\prime}, b_{5}, b_{6}^{\prime}, b_{7}, b_{8}, b_{9}, c_{1}^{\prime}, c_{2}, c_{3}, c_{4}, c_{5}, d^{\prime}\right\}
$$

of the algebra $\mathscr{L}(\beta, 0)$, where

$$
\begin{aligned}
& b_{1}^{\prime}=b_{1}+\beta^{2} b_{9} \\
& b_{3}^{\prime}=b_{3}+\beta^{2} b_{8} \\
& b_{4}^{\prime}=b_{4}+\beta^{2} c_{3} \\
& b_{6}^{\prime}=b_{6}+\beta^{2} c_{2} \\
& c_{1}^{\prime}=b_{1}+\beta^{2} c_{5} \\
& d^{\prime}=d+\beta^{2} b_{5} .
\end{aligned}
$$

It is straightforward to check that in this basis the multiplication table coincides with the multiplication table (3) with $\beta=\delta=0$.

An immediate corollary of these two lemmas is

Theorem 1. For any $\beta, \delta \in K$, we have $\mathscr{L}(\beta, \delta) \simeq \mathscr{L}(0,0)$.

Since the algebra $\mathscr{L}(0,0)$ is isomorphic to the smallest 15 -dimensional algebra in the family of simple Lie algebras constructed by Skryabin in [Sk, pp.691-692], we will refer to it in the rest of the paper as the Skryabin algebra. The rest of the paper is devoted to elucidation of some properties, and computation of some invariants of the Skryabin algebra.

\section{2-ENVELOPE, DERIVATIONS, SANDWICH SUBALGEBRA}

We continue to employ the basis $\left\{b_{1}, \ldots, b_{9}, c_{1}, \ldots, c_{5}, d\right\}$ of the Skryabin algebra $\mathscr{L}$ from the previous section (referred as the standard basis in what follows). The multiplication table is given by (3), where $\beta=\delta=0$.

The Skryabin algebra is not a 2 -algebra; its 2 -envelope $\mathscr{L}_{2}$ has dimension 19 , with additional basis elements $b_{1}^{[2]}, b_{4}^{[2]}, b_{7}^{[2]}, c_{3}^{[2]}$, the 2-map:

$$
\begin{gathered}
b_{2}^{[2]}=c_{4}, \quad b_{3}^{[2]}=b_{3}, \quad c_{1}^{[2]}=b_{9}, \quad d^{[2]}=b_{4}^{[2]}, \\
b_{5}^{[2]}=b_{6}^{[2]}=b_{8}^{[2]}=b_{9}^{[2]}=c_{2}^{[2]}=c_{4}^{[2]}=c_{5}^{[2]}=0, \\
b_{7}^{[4]}=b_{7}^{[2]}, \quad b_{1}^{[4]}=b_{4}^{[4]}=c_{3}^{[4]}=0,
\end{gathered}
$$


and the multiplication:

\begin{tabular}{|c||c|c|c|c|}
\hline & $b_{1}^{[2]}$ & $b_{4}^{[2]}$ & $b_{7}^{[2]}$ & $c_{3}^{[2]}$ \\
\hline \hline$b_{1}$ & 0 & 0 & 0 & $b_{8}$ \\
\hline$b_{2}$ & $b_{1}$ & 0 & 0 & 0 \\
\hline$b_{3}$ & 0 & 0 & 0 & 0 \\
\hline$b_{4}$ & 0 & 0 & $b_{4}$ & $c_{2}$ \\
\hline$b_{5}$ & $b_{4}$ & 0 & $b_{5}$ & 0 \\
\hline$b_{6}$ & 0 & 0 & $b_{6}$ & 0 \\
\hline$b_{7}$ & 0 & $b_{1}$ & 0 & 0 \\
\hline$b_{8}$ & $b_{7}$ & $b_{2}$ & 0 & 0 \\
\hline$b_{9}$ & 0 & $b_{3}$ & 0 & 0 \\
\hline$c_{1}$ & 0 & $b_{4}$ & $c_{1}$ & 0 \\
\hline$c_{2}$ & $c_{1}$ & $b_{5}$ & $c_{2}$ & 0 \\
\hline$c_{3}$ & $d$ & $b_{6}$ & $c_{3}$ & 0 \\
\hline$c_{4}$ & $b_{3}$ & 0 & 0 & 0 \\
\hline$c_{5}$ & $b_{6}$ & 0 & $c_{5}$ & 0 \\
\hline$d$ & 0 & 0 & $d$ & $c_{5}$ \\
\hline
\end{tabular}

\begin{tabular}{|l||c|c|c|}
\hline & $b_{4}^{[2]}$ & $b_{7}^{[2]}$ & $c_{3}^{[2]}$ \\
\hline \hline$b_{1}^{[2]}$ & 0 & 0 & $b_{9}$ \\
\hline$b_{4}^{\lfloor 2]}$ & & 0 & $c_{4}$ \\
\hline$b_{7}^{\lfloor 2]}$ & & & 0 \\
\hline
\end{tabular}

In what follows, we will employ the following standard notation for arbitrary elements of $\mathscr{L}$ :

$$
\lambda_{1} b_{1}+\cdots+\lambda_{9} b_{9}+\mu_{1} c_{1}+\cdots+\mu_{5} c_{5}+\eta d,
$$

and of $\mathscr{L}_{2}$ :

(4)

$$
\lambda_{1} b_{1}+\cdots+\lambda_{9} b_{9}+\mu_{1} c_{1}+\cdots+\mu_{5} c_{5}+\eta d+\xi_{1} b_{1}^{[2]}+\xi_{4} b_{4}^{[2]}+\xi_{7} b_{7}^{[2]}+\xi_{3} c_{3}^{[2]},
$$

where $\lambda_{1}, \ldots, \lambda_{9}, \mu_{1}, \ldots, \mu_{5}, \eta, \xi_{1}, \xi_{4}, \xi_{7}, \xi_{3} \in K$.

Proposition 1. The derivation algebra of the Skryabin algebra coincides with its 2-envelope.

Proof. Straightforward calculations.

Recall that an element $x$ of a Lie algebra $L$ is called a sandwich if $(\operatorname{ad} x)^{2}=0$ and $[[L, x],[L, x]]=$ 0 . (It is well-known - and easy to see - that if the characteristic of the ground field is different from 2 , then the second condition follows from the first one, but in characteristic 2 this is not true). The set of all sandwiches is multiplicatively closed, what implies that the sandwich subalgebra, i.e., the subalgebra of $L$ generated by all sandwiches, is just the linear span of sandwiches.

It follows from the result first proved by Kostrikin and Zelmanov, that a Lie algebra generated by sandwiches is nilpotent. See [V-L1, §3.2] for details and further references.

More generally, we will call a derivation $D$ of a Lie algebra $L$ a sandwich derivation, if $D^{2}=0$ and $[D(L), D(L)]=0$.

Lemma 3. The set of elements $x \in \mathscr{L}_{2}$ such that

$$
[[\mathscr{L}, x],[\mathscr{L}, x]]=0
$$

coincides with the linear span of elements $c_{2}, c_{4}, c_{5}, c_{3}^{[2]}$.

Proof. It is a matter of straightforward verification that any linear span of elements $c_{2}, c_{4}, c_{5}$, $c_{3}^{[2]}$ satisfies the condition (5).

Conversely, let $x$ be an arbitrary element (4) of $\mathscr{L}_{2}$ satisfying this condition. We perform the following calculations:

- Collecting in the equality $\left[\left[x, b_{8}\right],\left[x, c_{5}\right]\right]=0$ the terms containing $c_{1}$ and $c_{2}$, we get, respectively, $\xi_{1}^{2}=0$ and $\lambda_{1}^{2}+\xi_{1} \xi_{7}=0$, whence $\xi_{1}=0$ and $\lambda_{1}=0$.

- Collecting in the equality $\left[\left[x, b_{6}\right],\left[x, c_{4}\right]\right]=0$ the terms containing $c_{5}$, we get $\lambda_{7}^{2}=0$, whence $\lambda_{7}=0$. 
- Collecting in the equality $\left[\left[x, b_{1}\right],\left[x, c_{4}\right]\right]=0$ the terms containing $b_{8}$, we get $\mu_{1}^{2}=0$, whence $\mu_{1}=0$.

- Collecting in the equality $\left[\left[x, b_{6}\right],\left[x, c_{2}\right]\right]=0$ the terms containing $b_{8}$, we get $\eta^{2}=0$, whence $\eta=0$.

- Collecting in the equality $\left[\left[x, b_{8}\right],\left[x, c_{3}\right]\right]=0$ the terms containing $b_{5}$ and $c_{2}$, we get, respectively, $\xi_{4}^{2}=0$ and $\lambda_{4}^{2}+\lambda_{3} \xi_{4}=0$, whence $\xi_{4}=0$ and $\lambda_{4}=0$.

- Collecting in the equality $\left[\left[x, b_{1}\right],\left[x, b_{8}\right]\right]=0$ the terms containing $b_{9}$ and $c_{4}$, we get, respectively, $\lambda_{3}^{2}=0$ and $\lambda_{6}^{2}=0$, whence $\lambda_{3}=0$ and $\lambda_{6}=0$.

- Collecting in the equality $\left[\left[x, b_{4}\right],\left[x, c_{2}\right]\right]=0$ the terms containing $c_{4}$, we get $\xi_{7}^{2}=0$, whence $\xi_{7}=0$.

- Collecting in the equality $\left[\left[x, b_{1}\right],\left[x, b_{6}\right]\right]=0$ the terms containing $b_{5}$ and $c_{2}$, we get, respectively, $\lambda_{2}^{2}=0$ and $\mu_{3}^{2}=0$, whence $\lambda_{2}=0$ and $\mu_{3}=0$.

- Collecting in the equality $\left[\left[x, b_{1}\right],\left[x, c_{3}\right]\right]=0$ the terms containing $c_{2}$, we get $\lambda_{9}^{2}=0$, whence $\lambda_{9}=0$.

- Collecting in the equality $\left[\left[x, b_{1}\right],\left[x, b_{4}\right]\right]=0$ the terms containing $c_{5}$, we get $\lambda_{8}^{2}=0$, whence $\lambda_{8}=0$.

- Collecting in the equality $\left[\left[x, b_{1}\right],\left[x, b_{7}\right]\right]=0$ the terms containing $c_{4}$, we get $\lambda_{5}^{2}=0$, whence $\lambda_{5}=0$.

We are left with a linear combination of $c_{2}, c_{4}, c_{5}, c_{3}^{[2]}$.

As an immediate corollary of this lemma, we have

\section{Proposition 2.}

(i) The sandwich subalgebra of $\mathscr{L}$ is 3-dimensional abelian, linearly spanned by elements $c_{2}$, $c_{4}, c_{5}$.

(ii) The set of sandwich derivations of $\mathscr{L}$ forms a 4-dimensional abelian subalgebra of $\mathscr{L}_{2}$ spanned by the inner derivations ad $c_{2}$, ad $c_{4}$, ad $c_{5}$, and by the outer derivation $\operatorname{ad} c_{3}^{[2]}$.

Proof. (i) By Lemma 3 , the sandwich subalgebra of $\mathscr{L}$ lies in the linear span of $c_{2}, c_{4}, c_{5}$. Since these elements pairwise commute, they span the 3-dimensional abelian subalgebra of $\mathscr{L}$, and

$$
\left(\mu_{1} c_{2}+\mu_{4} c_{4}+\mu_{5} c_{5}\right)^{[2]}=\mu_{1}^{2} c_{2}^{[2]}+\mu_{4}^{2} c_{4}^{[2]}+\mu_{5}^{2} c_{5}^{[2]}=0,
$$

thus any element in this 3-dimensional subalgebra satisfies the condition $(\operatorname{ad} x)^{2}=0$.

(ii) By Proposition 1, any derivation is an element of $\mathscr{L}_{2}$. Apply Lemma 3 and reason as above.

Note that from Lemma 3 and Proposition 2 it follows that in the Skryabin algebra $\mathscr{L}$, the condition $[[\mathscr{L}, x],[\mathscr{L}, x]]=0$ implies $[[\mathscr{L}, x], x]=0$. In general, this is, of course, not true.

\section{Computations over GF(2), The ABSolute toral RANK, THin DECOMPOSition}

3.1. Some numerology. In this section we report on computations over GF(2), performed on the computer in GAP, [G]. A brute-force search on the computer shows that in the 2-envelope of the Skryabin algebra $\mathscr{L}$ over GF(2) there are 384 toral elements, 6144 2-dimensional tori, 21504 3-dimensional tori, 26880 4-dimensional tori, and no 5-dimensional tori (no attempt was made to determine their conjugacy classes with respect to the automorphism group).

The centralizer in $\mathscr{L}$ of each of the 384 toral elements is 7-dimensional, and for 240 toral elements the centralizer is (central) simple. Among these 240 simple algebras, 48 have absolute toral rank 2, and 192 have absolute toral rank 3. As proved in [V-L2, Theorem 1] (and confirmed by computations in [E]), over GF(2) there exist two simple 7-dimensional Lie algebras. These algebras are identified as (forms) of the Zassenhaus algebra $W_{1}^{\prime}(3)$, and the Hamiltonian algebra $H_{2}^{\prime \prime}\left((2,1),\left(1+x_{1}^{(3)} x_{2}\right) d x_{1} \wedge d x_{2}\right)$, denoted by us here simply as $W$ and $H$, respectively (see [GG] and

\footnotetext{
${ }^{\dagger}$ The GAP code is available at https : //web.osu.cz/ Zusmanovich/papers/15dim/.
} 
[GGA] for further info, including explicit multiplication tables of these algebras, and their identification with some other simple 7-dimensional Lie algebras from the literature). Both algebras have absolute toral rank 3 over an algebraically closed field, but over GF(2) $W$ has absolute toral rank 2 , while $H$ has absolute toral rank 3; thus the absolute toral rank can be used to distinguish them as subalgebras of the Skryabin algebra in our computations.

3.2. Some examples. Let us exhibit explicitly one of the maximal tori, and one of the 7-dimensional simple subalgebras mentioned in the previous subsection.

Here is just one of the 4-dimensional tori, linearly spanned by the toral elements

$$
b_{1}+b_{3}+b_{1}^{[2]}, \quad b_{2}+b_{3}+c_{4}+b_{7}^{[2]}, \quad b_{4}+b_{6}+b_{4}^{[2]}+b_{7}^{[2]}, \quad b_{8}+b_{9}+c_{1}+c_{3}+b_{7}^{[2]}+c_{3}^{[2]} .
$$

Now take the first toral element in this torus, $h=b_{1}+b_{3}+b_{1}^{[2]}$. Its centralizer $C_{\mathscr{L}}(h)$ has the basis

$$
b_{1}+b_{3}, \quad b_{2}+c_{3}+c_{4}, \quad b_{4}+b_{6}, \quad b_{5}+b_{6}+c_{5}, \quad b_{7}+b_{9}, \quad c_{1}+c_{3}, \quad d .
$$

It is straightforward to check that in this basis the multiplication table of $C_{\mathscr{L}}(h)$ coincides with the multiplication table of the simple 7-dimensional Hamiltonian algebra $H$ (see [GG, §3] or [GGA, §1]) via the identification

$$
\begin{gathered}
V_{0} \leftrightarrow b_{5}+b_{6}+c_{5}, \quad V_{1} \leftrightarrow c_{1}+c_{3}, \quad E_{1} \leftrightarrow b_{7}+b_{9}, \quad E_{0} \leftrightarrow b_{2}+c_{3}+c_{4}, \\
F_{1} \leftrightarrow b_{4}+b_{6}, \quad F_{0} \leftrightarrow d, \quad G \leftrightarrow b_{1}+b_{3} .
\end{gathered}
$$

3.3. The absolute toral rank. The computer calculation in $\$ 3.1$ shows that the absolute toral rank of the Skryabin algebra over GF(2) is equal to 4 . However, a bit of additional work allows to establish this result over an arbitrary field.

Theorem 2. The absolute toral rank of the Skryabin algebra is equal to 4.

Proof. As we want to establish this result over an arbitrary field $K$, we will distinguish the Skryabin algebra $\overline{\mathscr{L}}=\mathscr{L} \otimes_{\mathrm{GF}(2)} K$ over $K$, and its $\mathrm{GF}(2)$-form $\mathscr{L}$.

A direct computer verification shows that each of the 268804 -dimensional tori $T$ in $\mathscr{L}_{2}$ coincides with its normalizer, i.e., is a Cartan subalgebra of $\mathscr{L}_{2}$. Consequently, $\bar{T}=T \otimes_{\mathrm{GF}(2)} K$ is a Cartan subalgebra of $\overline{\mathscr{L}_{2}}=(\overline{\mathscr{L}})_{2}$. By [P, Theorem 2(ii)]], $\bar{T}$ is a torus of the maximal possible dimension in $\overline{\mathscr{L}}_{2}$, and hence the absolute toral rank of $\overline{\mathscr{L}}$ is equal to 4 .

Alternatively, a direct simple proof free from reference to the computer can be provided by just looking at one of the 4-dimensional tori, for example, at (6). Indeed, a direct calculation shows that (6) coincides with its normalizer in the 2-envelope of any Skryabin algebra $\overline{\mathscr{L}}$, and hence by the same reference to $[\overline{\mathrm{P}}]$ is a torus of the maximal possible dimension.

3.4. Thin decomposition. Let us consider the following situation. Assume that a Lie algebra $L$ has dimension $2^{n}-1$, the absolute toral rank of $L$ is $n, T$ is a torus in the 2-envelope of $L$ of (the maximal) dimension $n$, and $T \cap L=0$. Assume further that the roots of the action of $T$ on $L$ are exactly nonzero tuples in GF(2) (in particular, the centralizer of $T$ in $L$ is zero), and each root space is one-dimensional. Thus, the root space decomposition is of the form

$$
L=\bigoplus_{\substack{\alpha \in \mathrm{GF}(2)^{n} \\ \alpha \neq(0, \ldots, 0)}} K e_{\alpha}
$$

for some elements $e_{\alpha} \in L$. In particular, the multiplication table of $L$ in the basis $\left\{e_{\alpha} \mid \alpha \in\right.$ $\left.\mathrm{GF}(2)^{n} \backslash(0, \ldots, 0)\right\}$ has the following form: either $\left[e_{\alpha}, e_{\beta}\right]=e_{\alpha+\beta}$, or $\left[e_{\alpha}, e_{\beta}\right]=0$.

In such a situation, we will call the decomposition (7) thin. Some simple Lie algebras (for example, the 7-dimensional algebras, as shown in [GG]) admit a thin decomposition. We suggest that this is an important property of simple Lie algebras which should be taken into account in the classification efforts. 
A direct computer verification shows that the Skryabin algebra admits a thin decomposition with respect to each of the 268804 -dimensional tori in its 2 -envelope. We will provide explicitly one of them, corresponding to the torus (6).

The corresponding generators of the one-dimensional root spaces are:

$$
\begin{aligned}
& e_{0001}=b_{2}+b_{3}+b_{4}+b_{6}+c_{4} \\
& e_{0010}=b_{2}+b_{3}+c_{1}+c_{3}+c_{4} \\
& e_{0011}=b_{2}+b_{3}+b_{4}+b_{6}+c_{1}+c_{3}+c_{4} \\
& e_{0100}=b_{1}+b_{3}+b_{7}+b_{9}+d \\
& e_{0101}=b_{7}+b_{9}+d \\
& e_{0110}=b_{1}+b_{3}+b_{5}+b_{6}+c_{5}+d \\
& e_{0111}=b_{5}+b_{6}+c_{5} \\
& e_{1000}=b_{2}+b_{3}+b_{8}+b_{9}+c_{1}+c_{2}+c_{3} \\
& e_{1001}=b_{2}+b_{3}+b_{4}+b_{5}+b_{6} \\
& e_{1010}=b_{2}+b_{3}+c_{1}+c_{2}+c_{3} \\
& e_{1011}=b_{2}+b_{3}+b_{4}+b_{5}+b_{6}+c_{1}+c_{2}+c_{3} \\
& e_{1100}=b_{1}+b_{2}+b_{3}+b_{7}+b_{8}+b_{9}+c_{2}+c_{3}+d \\
& e_{1101}=b_{5}+b_{6}+b_{7}+b_{8}+b_{9}+c_{2}+c_{3}+d \\
& e_{1110}=b_{1}+b_{2}+b_{3}+b_{5}+b_{6}+c_{2}+c_{3}+d \\
& e_{1111}=b_{5}+b_{6}
\end{aligned}
$$

and the corresponding multiplication table of $\mathscr{L}$ reads:

\begin{tabular}{|c||c|c|c|c|c|c|c|c|c|c|c|c|c|c|}
\hline & $e_{0010}$ & $e_{0011}$ & $e_{0100}$ & $e_{0101}$ & $e_{0110}$ & $e_{0111}$ & $e_{1000}$ & $e_{1001}$ & $e_{1010}$ & $e_{1011}$ & $e_{1100}$ & $e_{1101}$ & $e_{1110}$ & $e_{1111}$ \\
\hline \hline$e_{0001}$ & $e_{0011}$ & $e_{0010}$ & $e_{0101}$ & $e_{0100}$ & 0 & 0 & $e_{1001}$ & 0 & $e_{1011}$ & $e_{1010}$ & $e_{1101}$ & $e_{1100}$ & $e_{1111}$ & 0 \\
\hline$e_{0010}$ & & $e_{0001}$ & $e_{0110}$ & $e_{0111}$ & $e_{0100}$ & 0 & 0 & $e_{1011}$ & 0 & $e_{1001}$ & $e_{1110}$ & $e_{1111}$ & $e_{1100}$ & 0 \\
\hline$e_{0011}$ & & & $e_{0111}$ & $e_{0110}$ & $e_{0101}$ & 0 & $e_{1011}$ & $e_{1010}$ & $e_{1001}$ & 0 & 0 & $e_{1110}$ & $e_{1101}$ & 0 \\
\hline$e_{0100}$ & & & & 0 & $e_{0010}$ & $e_{0011}$ & $e_{1100}$ & $e_{1101}$ & $e_{1110}$ & 0 & $e_{1000}$ & $e_{1001}$ & 0 & $e_{1011}$ \\
\hline$e_{0101}$ & & & & & $e_{0011}$ & $e_{0010}$ & 0 & $e_{1100}$ & $e_{1111}$ & $e_{1110}$ & 0 & $e_{1000}$ & $e_{1011}$ & $e_{1010}$ \\
\hline$e_{0110}$ & & & & & & $e_{0001}$ & $e_{1110}$ & $e_{1111}$ & $e_{1100}$ & $e_{1101}$ & 0 & 0 & $e_{1000}$ & $e_{1001}$ \\
\hline$e_{0111}$ & & & & & & & 0 & 0 & 0 & 0 & $e_{1011}$ & $e_{1010}$ & $e_{1001}$ & 0 \\
\hline$e_{1000}$ & & & & & & & & $e_{0001}$ & 0 & $e_{0011}$ & $e_{0100}$ & 0 & $e_{0110}$ & 0 \\
\hline$e_{1001}$ & & & & & & & & & $e_{0011}$ & $e_{0010}$ & $e_{0101}$ & $e_{0100}$ & 0 & 0 \\
\hline$e_{1010}$ & & & & & & & & & & $e_{0001}$ & $e_{0110}$ & $e_{0111}$ & $e_{0100}$ & 0 \\
\hline$e_{1011}$ & & & & & & & & & & & $e_{0111}$ & $e_{0110}$ & $e_{0101}$ & 0 \\
\hline$e_{1100}$ & & & & & & & & & & & & $e_{0001}$ & $e_{0010}$ & $e_{0011}$ \\
\hline$e_{1101}$ & & & & & & & & & & & & & 0 & $e_{0010}$ \\
\hline$e_{1110}$ & & & & & & & & & & & & & & $e_{0001}$ \\
\hline
\end{tabular}

\section{THE AUTOMORPHISM GROUP}

The goal of this section is to determine the automorphism group of the Skryabin algebra. First we define three types of automorphisms - exponential automorphisms, a certain explicitly defined three one-parameter families, and diagonal automorphisms, determine the group generated by them, and then prove that they exhaust the whole automorphism group.

4.1. Exponential automorphisms. If $D$ is a sandwich derivation of a Lie algebra $L$, then $\exp (D)=1+D$ is an automorphism of $L$, called an exponential automorphism. If $D=\operatorname{ad} x$ is an inner derivation (i.e., $x$ is a sandwich in $L$ ), we will use the shorthand notation $\exp (x)$ instead of $\exp (\operatorname{ad} x)$.

Since $\exp (D)^{2}=\exp (2 D)=1$, exponential automorphisms are of order 2, and generate a unipotent subgroup of exponent 2, denoted by $\operatorname{Exp}(L)$, of the automorphism group $\operatorname{Aut}(L)$. Note also 
that if $D_{1}, D_{2} \in L$ are two commuting sandwich derivations, then the corresponding automorphisms also commute: $\exp \left(D_{1}\right) \circ \exp \left(D_{2}\right)=\exp \left(D_{2}\right) \circ \exp \left(D_{1}\right)$.

According to Proposition 2 (ii), the group $\operatorname{Exp}(\mathscr{L})$ is 4-dimensional abelian, isomorphic to the additive group $K^{4}=K \oplus K \oplus K \oplus K$. Let us write down its one-parameter generators explicitly (here and below we indicate only those basis elements, on which the automorphism acts nonidentically):

$$
\begin{aligned}
& b_{1} \mapsto b_{1}+\alpha c_{3} \\
& b_{3} \mapsto b_{3}+\alpha c_{2} \\
& \exp \left(\alpha c_{2}\right): b_{4} \mapsto b_{4}+\alpha c_{4} \\
& b_{7} \mapsto b_{7}+\alpha c_{5} \\
& d \mapsto d+\alpha b_{8} \\
& \exp \left(\alpha c_{4}\right): \begin{array}{l}
b_{1} \mapsto b_{1}+\alpha b_{2} \\
b_{4} \mapsto b_{4}+\alpha b_{5} \\
b_{7} \mapsto b_{7}+\alpha b_{8} \\
c_{1} \mapsto c_{1}+\alpha c_{2}
\end{array} \\
& b_{1} \mapsto b_{1}+\alpha b_{5} \\
& \exp \left(\alpha c_{5}\right): b_{7} \mapsto b_{7}+\alpha c_{2} \\
& d \mapsto d+\alpha c_{4} \\
& \begin{aligned}
\exp \left(\alpha c_{3}^{[2]}\right): & b_{1} \mapsto b_{1}+\alpha b_{8} \\
b_{4} & \mapsto b_{4}+\alpha c_{2}
\end{aligned} \\
& d \mapsto d+\alpha c_{5} \text {. }
\end{aligned}
$$

Here $\alpha \in K$ is a parameter.

4.2. On $\exp \left(\alpha c_{4}\right)$. For a moment, let us return to the realization of $\mathscr{L}$ as a filtered deformation of the semisimple Lie algebra (1). The exponential automorphisms $\exp \left(\alpha c_{4}\right)$ are the only automorphisms of $\mathscr{L}$ which are "lifted" from automorphisms of the algebra (1). Using the results about automorphisms of the tensor product of a simple Lie algebra and a divided power algebra (see, for example, [W, §2.2]), it is not difficult to describe the automorphism group of (1) (roughly, those are automorphisms of $S \otimes \mathcal{O}_{1}(2)$ invariant under the action of $g$ and of $\partial$ ). Automorphisms which are preserved by the cocycles defining the deformation, will be also automorphisms of $\mathscr{L}$. Among the automorphisms of (1), the only automorphisms satisfying this condition, are automorphisms acting on $\mathrm{S} \otimes \mathcal{O}_{1}(2)$ as $\phi(\alpha) \otimes \operatorname{id}_{\mathcal{O}_{1}(2)}$, where $\phi(\alpha)$ is an automorphism of $\mathrm{S}$ of the form

$$
e \mapsto e+\alpha f, \quad f \mapsto f, \quad h \mapsto h,
$$

and leaving $g \otimes\langle 1, x\rangle$ and $\partial$ invariant. In terms of the standard basis of $\mathscr{L}$, this is exactly $\exp \left(\alpha c_{4}\right)$. In general, the automorphism group of the algebra (1) is much smaller than the automorphism group of its deformation $\mathscr{L}$, which shows that, generally, there is no strong relationship between automorphisms of a Lie algebra and of its deformation.

4.3. Automorphisms $\Phi, \Psi$, and $\Theta$. Consider the following three one-parameter families of linear maps on $\mathscr{L}$, depending on the parameter $\alpha \in K$ :

$$
\begin{aligned}
& b_{1} \mapsto b_{1}+\alpha b_{4} \\
& b_{2} \mapsto b_{2}+\alpha b_{5} \\
& b_{7} \mapsto b_{7}+\alpha^{2} b_{2}+\alpha^{3} b_{5}+\alpha c_{1} \\
& b_{8} \mapsto b_{8}+\alpha c_{2} \\
& b_{9} \mapsto b_{9}+\alpha^{2} c_{4} \\
& c_{1} \mapsto c_{1}+\alpha^{2} b_{5} \\
& c_{3} \mapsto c_{3}+\alpha c_{4} \\
& d \mapsto d+\alpha b_{3}+\alpha^{2} b_{6}
\end{aligned}
$$




$$
\begin{aligned}
& b_{1} \mapsto b_{1}+\alpha b_{6}+\alpha c_{1}+\alpha^{2} c_{4} \\
& b_{2} \mapsto b_{2}+\alpha c_{2} \\
& b_{3} \mapsto b_{3}+\alpha b_{5} \\
& b_{4} \mapsto b_{4}+\alpha b_{2}+\alpha^{2} c_{2} \\
& \Psi(\alpha): b_{6} \mapsto b_{6}+\alpha c_{4} \\
& b_{7} \mapsto b_{7}+\alpha b_{5}+\alpha c_{3} \\
& b_{9} \mapsto b_{9}+\alpha c_{2}+\alpha c_{5} \\
& c_{1} \mapsto c_{1}+\alpha b_{8}+\alpha c_{4} \\
& d \mapsto d+\alpha b_{2}+\alpha b_{9}+\alpha^{2} c_{2}+\alpha^{2} c_{5} \\
& b_{1} \mapsto b_{1}+\alpha b_{7}+\alpha^{3} b_{8}+\alpha^{2} b_{9} \\
& b_{2} \mapsto b_{2}+\alpha b_{8} \\
& b_{3} \mapsto b_{3}+\alpha^{2} b_{8} \\
& b_{4} \mapsto b_{4}+\alpha^{2} b_{5}+\alpha c_{1}+\alpha^{2} c_{3}+\alpha^{3} c_{5} \\
& \Theta(\alpha): b_{5} \mapsto b_{5}+\alpha c_{2} \\
& b_{6} \mapsto b_{6}+\alpha^{2} c_{2}+\alpha^{2} c_{5} \\
& c_{1} \mapsto c_{1}+\alpha^{2} c_{2}+\alpha^{2} c_{5} \\
& c_{3} \mapsto c_{3}+\alpha c_{5} \\
& d \mapsto d+\alpha^{2} b_{5}+\alpha b_{6}+\alpha^{3} c_{2}+\alpha^{2} c_{3}
\end{aligned}
$$

Direct calculations show that all of them are automorphisms of $\mathscr{L}$, and

$$
\begin{aligned}
& \Phi(\alpha) \circ \Phi\left(\alpha^{\prime}\right)=\Phi\left(\alpha+\alpha^{\prime}\right) \\
& \Psi(\alpha) \circ \Psi\left(\alpha^{\prime}\right)=\Psi\left(\alpha+\alpha^{\prime}\right) \circ \exp \left(\alpha \alpha^{\prime} c_{3}^{[2]}\right) \\
& \Theta(\alpha) \circ \Theta\left(\alpha^{\prime}\right)=\Theta\left(\alpha+\alpha^{\prime}\right) \circ \exp \left(\left(\alpha^{2} \alpha^{\prime}+\alpha \alpha^{\prime 2}\right) c_{3}^{[2]}\right)
\end{aligned}
$$

for any $\alpha, \alpha^{\prime} \in K$. In particular, the automorphisms $\Phi(\alpha)$ and $\Theta(\alpha)$ are of order 2 , and the automorphisms $\Psi(\alpha)$ are of order 4 .

4.4. Diagonal automorphisms. Let $L$ be a Lie algebra with a basis $B$. We will call an automorphism of $L$ diagonal with respect to $B$ (or just diagonal if it is clear which basis $B$ is meant), if it leaves invariant each one-dimensional subspace $K x, x \in B$.

Lemma 4. Each diagonal automorphism of $\mathscr{L}$ with respect to the standard basis is of the form

$$
\begin{array}{rlrl}
b_{1} & \mapsto \lambda^{-2} b_{1} & c_{1} \mapsto \lambda c_{1} \\
b_{2} \mapsto \lambda^{2} b_{2} & c_{2} \mapsto \lambda^{5} c_{2} \\
b_{3} \mapsto b_{3} & c_{3} \mapsto \lambda^{3} c_{3} \\
b_{4} \mapsto \lambda^{-1} b_{4} & c_{4} \mapsto \lambda^{4} c_{4} \\
\Delta(\lambda): & b_{5} \mapsto \lambda^{3} b_{5} & c_{5} \mapsto \lambda^{5} c_{5} \\
& b_{6} \mapsto \lambda b_{6} & d \mapsto \lambda^{-1} d \\
b_{7} \mapsto b_{7} & \\
b_{8} \mapsto \lambda^{4} b_{8} & \\
b_{9} \mapsto \lambda^{2} b_{9} &
\end{array}
$$

where $\lambda \in K^{*}$.

Proof. Let $x \mapsto \alpha(x) x$, where $x$ is an element in the standard basis, be a diagonal automorphism of $\mathscr{L}$. Denote $\alpha\left(b_{4}\right)=\lambda^{-1}$.

We perform the following calculations:

- $\alpha\left(b_{3}\right)=\alpha\left(b_{1}\right) \alpha\left(b_{2}\right), \alpha\left(b_{1}\right)=\alpha\left(b_{1}\right) \alpha\left(b_{3}\right)$, and $\alpha\left(b_{2}\right)=\alpha\left(b_{2}\right) \alpha\left(b_{3}\right)$ imply $\alpha\left(b_{2}\right)=\alpha\left(b_{1}\right)^{-1}$ and $\alpha\left(b_{3}\right)=1$.

- $\alpha\left(b_{1}\right)=\alpha\left(b_{4}\right) \alpha(d)$ and $\alpha(d)=\alpha\left(b_{1}\right) \alpha\left(c_{1}\right)$ imply $\alpha\left(c_{1}\right)=\lambda$.

- $\alpha\left(b_{7}\right)=\alpha\left(c_{1}\right) \alpha(d)=\alpha\left(b_{1}\right) \lambda^{2}$. 
- $b_{7}^{[4]}=b_{7}^{[2]}$ implies $\alpha\left(b_{7}\right)^{4}=\alpha\left(b_{7}\right)^{2}$, thus $\alpha\left(b_{7}\right)^{2}=1$ and $\alpha\left(b_{7}\right)=1, \alpha\left(b_{1}\right)=\lambda^{-2}, \alpha\left(b_{2}\right)=\lambda^{2}$, and $\alpha(d)=\lambda^{-1}$.

- $\alpha\left(c_{3}\right)=\alpha\left(b_{1}\right) \alpha\left(c_{2}\right)=\alpha\left(c_{2}\right) \lambda^{-2}$.

- $\alpha\left(c_{1}\right)=\alpha\left(b_{1}\right) \alpha\left(c_{3}\right)$ implies $\lambda=\alpha\left(c_{2}\right) \lambda^{-4}$, thus $\alpha\left(c_{2}\right)=\lambda^{5}$, and $\alpha\left(c_{3}\right)=\lambda^{3}$.

- $\alpha\left(b_{2}\right)=\alpha\left(b_{1}\right) \alpha\left(c_{4}\right)$ implies $\alpha\left(c_{4}\right)=\lambda^{4}$.

- $\alpha\left(b_{9}\right)=\alpha\left(b_{2}\right) \alpha\left(b_{7}\right)=\lambda^{2}$.

- $\alpha\left(b_{8}\right)=\alpha\left(b_{2}\right) \alpha\left(b_{9}\right)=\lambda^{4}$.

- $\alpha\left(c_{2}\right)=\alpha\left(b_{7}\right) \alpha\left(c_{5}\right)$ implies $\alpha\left(c_{5}\right)=\lambda^{5}$.

- $\alpha\left(b_{5}\right)=\alpha\left(b_{4}\right) \alpha\left(c_{4}\right)=\lambda^{3}$.

- $\alpha\left(b_{6}\right)=\alpha\left(b_{1}\right) \alpha\left(b_{5}\right)=\lambda$.

Conversely, it is straightforward to verify that each map of the form (9) is an automorphism of $\mathscr{L}$. Therefore, the group of diagonal automorphisms $\operatorname{Diag}(\mathscr{L})$ is isomorphic to $K^{*}$, the multiplicative group of $K$.

4.5. Putting all this together. Denote by $\operatorname{Aut}_{0}(\mathscr{L})$ the group generated by the just defined automorphisms of $\mathscr{L}$.

Direct calculations show that for each $\alpha, \gamma \in K$, any of $\Phi(\alpha), \Psi(\alpha)$, and $\Theta(\alpha)$ commutes with any of $\exp \left(\gamma c_{2}\right), \exp \left(\gamma c_{4}\right), \exp \left(\gamma c_{5}\right)$, and $\exp \left(\gamma c_{3}^{[2]}\right)$. Additionally,

$$
\begin{aligned}
& \Psi(\gamma) \circ \Phi(\alpha)=\Phi(\alpha) \circ \Psi(\gamma) \circ \exp \left(\alpha \gamma c_{4}\right) \\
& \Theta(\gamma) \circ \Phi(\alpha)=\Phi(\alpha) \circ \Theta(\gamma) \circ \exp \left(\alpha \gamma^{2} c_{2}\right) \circ \exp \left(\alpha^{2} \gamma c_{4}\right) \circ \exp \left(\alpha \gamma^{2} c_{5}\right) \circ \exp \left(\alpha^{2} \gamma^{2} c_{3}^{[2]}\right) \\
& \Theta(\gamma) \circ \Psi(\alpha)=\Psi(\alpha) \circ \Theta(\gamma) \circ \exp \left(\alpha \gamma c_{2}\right) \circ \exp \left(\alpha \gamma c_{5}\right) .
\end{aligned}
$$

Together with (8) this imply that the group $\mathcal{N}$ generated by the exponential automorphisms, and automorphisms $\Phi, \Psi$ and $\Theta$, is a 7-dimensional unipotent algebraic group. Further, taking into account that $\Delta(\lambda)^{-1}=\Delta\left(\lambda^{-1}\right)$, we have:

$$
\begin{array}{ll}
\exp \left(\alpha c_{2}\right)^{\Delta(\lambda)} & =\exp \left(\lambda^{-5} \alpha c_{2}\right) \\
\exp \left(\alpha c_{4}\right)^{\Delta(\lambda)} & =\exp \left(\lambda^{-4} \alpha c_{4}\right) \\
\exp \left(\alpha c_{5}\right)^{\Delta(\lambda)} & =\exp \left(\lambda^{-5} \alpha c_{5}\right) \\
\exp \left(\alpha c_{3}^{[2]}\right)^{\Delta(\lambda)} & =\exp \left(\lambda^{-6} \alpha c_{3}^{[2]}\right) \\
\Phi(\alpha)^{\Delta(\lambda)} & =\Phi\left(\lambda^{-1} \alpha\right) \\
\Psi(\alpha)^{\Delta(\lambda)} & =\Psi\left(\lambda^{-3} \alpha\right) \\
\Theta(\alpha)^{\Delta(\lambda)} & =\Theta\left(\lambda^{-2} \alpha\right) .
\end{array}
$$

Therefore, $\mathcal{N}$ is a normal subgroup in $\operatorname{Aut}_{0}(\mathscr{L})$, and $\operatorname{Aut}_{0}(\mathscr{L})$ is isomorphic to the semidirect product $K^{*} \ltimes \mathcal{N}$, with the action of $K^{*}$ on $\mathcal{N}$ defined by (10).

4.6. Invariant subspaces. Now we are going to prove that the automorphisms constructed in the previous sections exhaust all automorphisms of $\mathscr{L}$. To this aim, we determine certain invariant subspaces in the Skryabin algebra. 
Proposition 3. The Skryabin algebra $\mathscr{L}$ possesses the following Aut(L्L)-invariant subspaces:

$$
\begin{gathered}
\left\langle c_{2}\right\rangle \quad\left\langle c_{4}\right\rangle \quad\left\langle c_{5}\right\rangle \\
\left\langle b_{5}, c_{2}\right\rangle \quad\left\langle b_{8}, c_{2}\right\rangle \\
V_{4}=\left\langle b_{2}, b_{5}, b_{8}, c_{2}\right\rangle \\
V_{5}=\left\langle b_{8}, c_{2}, c_{3}, c_{4}, c_{5}\right\rangle \\
V_{6}^{\prime}=\left\langle b_{5}, b_{6}, b_{8}, c_{2}, c_{4}, c_{5}\right\rangle \quad V_{6}^{\prime \prime}=\left\langle b_{5}, b_{8}, b_{9}, c_{2}, c_{4}, c_{5}\right\rangle \\
V_{6}=\left\langle b_{2}, b_{3}, b_{5}, b_{8}, c_{2}, c_{4}\right\rangle \quad b_{8},\left\langle b_{2}, b_{3}, b_{5}, b_{6}, b_{8}, c_{2}, c_{4}, c_{5}\right\rangle \quad V_{8}^{\prime}=\left\langle b_{2}, b_{5}, b_{6}, b_{8}, b_{9}, c_{2}, c_{4}, c_{5}\right\rangle \quad V_{8}^{\prime \prime}=\left\langle b_{2}, b_{5}, b_{6}, b_{8}, c_{2}, c_{3}, c_{4}, c_{5}\right\rangle \\
V_{7}=\left\langle b_{5}, b_{6}, b_{8}, b_{9}, c_{2}, c_{4}, c_{5}\right\rangle \quad V_{7}^{\prime}=\left\langle b_{5}, b_{8}, b_{9}, c_{2}, c_{3}, c_{4}, c_{5}\right\rangle \\
V_{9}=\left\langle b_{2}, b_{3}, b_{5}, b_{6}, b_{8}, b_{9}, c_{2}, c_{4}, c_{5}\right\rangle \quad V_{9}^{\prime}=\left\langle b_{2}, b_{5}, b_{6}, b_{8}, b_{9}, c_{2}, c_{3}, c_{4}, c_{5}\right\rangle \\
V_{11}=\left\langle b_{2}, b_{3}, b_{4}, b_{5}, b_{6}, b_{8}, c_{1}, \ldots, c_{5}\right\rangle \quad V_{11}^{\prime}=\left\langle b_{2}, b_{3}, b_{5}, b_{6}, b_{8}, b_{9}, c_{1}, \ldots, c_{5}\right\rangle \\
V_{11}^{\prime \prime}=\left\langle b_{2}, b_{3}, b_{5}, b_{6}, b_{8}, b_{9}, c_{2}, c_{3}, c_{4}, c_{5}, d\right\rangle \\
V_{12}=\left\langle b_{2}, b_{3}, b_{4}, b_{5}, b_{6}, b_{8}, b_{9}, c_{1}, \ldots, c_{5}\right\rangle
\end{gathered}
$$

Proof. By Proposition 2(1), the sandwich subalgebra $S$ of $\mathscr{L}$ coincides with $\left\langle c_{2}, c_{4}, c_{5}\right\rangle$. Starting from this, rewrite the specified subspaces in invariant terms:

- $\left\langle c_{5}\right\rangle=\{x \in S \mid \operatorname{dim}[\mathscr{L}, x] \leq 3\}$;

- $\left\langle c_{4}, c_{5}\right\rangle$ is the subspace (actually, the abelian subalgebra) linearly spanned by elements $x \in S$ such that $\operatorname{dim}[\mathscr{L}, x] \leq 4$

- $\left\langle c_{4}\right\rangle=\left[\mathscr{L}, c_{5}\right] \cap\left\langle c_{4}, c_{5}\right\rangle$

- $V_{4}=\left[\mathscr{L}, c_{4}\right]$

- $\left\langle c_{2}\right\rangle=S \cap V_{4}$

- $V_{5}=\left[\mathscr{L}, c_{2}\right]$

- $\left\langle b_{8}, c_{2}\right\rangle=V_{4} \cap V_{5}$

- $V_{7}^{\prime}=\left[\mathscr{L},\left\langle b_{8}, c_{2}\right\rangle\right]$

- $V_{9}^{\prime}=\{x \in \mathscr{L} \mid[x, S]=0\}$

- $\left\langle b_{5}, c_{2}\right\rangle=\left[\mathscr{L}, c_{5}\right] \cap V_{4} \cap\left[V_{9}^{\prime}, V_{9}^{\prime}\right]$

- $V_{8}^{\prime}=\left\{x \in V_{9}^{\prime} \mid x^{[2]} \in \mathscr{L}\right\}$;

- $V_{8}^{\prime \prime}=\left[\mathscr{L},\left\langle b_{5}, c_{2}\right\rangle\right]$

- Note that $V_{8}^{\prime}$ is a subalgebra of $\mathscr{L}$ with the center $S$. The subspace $V_{7}$ (actually, a subalgebra) coincides with the set of elements $x \in V_{8}^{\prime}$ such that the induced adjoint map ad $x: V_{8}^{\prime} / S \rightarrow V_{8}^{\prime} / S$ has rank $\leq 1$;

- $V_{6}^{\prime}=V_{7} \cap V_{8}^{\prime \prime}$

- $V_{6}^{\prime \prime}=V_{7} \cap V_{7}^{\prime}$

- $V_{11}^{\prime}=\{x \in \mathscr{L} \mid[x, S] \subseteq S\}$

- $V_{11}^{\prime \prime}=C_{\mathscr{L}}\left(c_{4}\right)$

- $V_{9}=\left[V_{11}^{\prime \prime}, V_{11}^{\prime \prime}\right]$;

- $V_{12}=\left\{x \in \mathscr{L} \mid\left[x, V_{11}^{\prime}\right] \subseteq V_{11}^{\prime}+K x\right\}$

- $V_{11}=\left[V_{12}, V_{12}\right]$

- $V_{8}=V_{9} \cap V_{11}$

- $V_{6}$ is a linear span of elements $x^{[2]}$, where $x \in V_{8}$;

All this is verified by straightforward computations.

Many more Aut( $\mathscr{L}$ )-invariant subspaces of $\mathscr{L}$ can be produced in a similar fashion, we confine here ourselves only to those which will be needed in the sequel.

\subsection{No other automorphisms.}

Theorem 3. Assume that any quadratic equation with coefficients in the ground field $K$ has a solution in $K$. Then $\operatorname{Aut}(\mathscr{L})=\operatorname{Aut}_{0}(\mathscr{L})$. 
Proof. Let $\varphi$ be an automorphism of $\mathscr{L}$. Our strategy is consecutively "twist" $\varphi$ by taking compositions with various automorphisms from $\operatorname{Aut}_{0}(\mathscr{L})$, and eventually arrive to the conclusion $\varphi=\mathrm{id} \mathscr{L}$.

By Proposition $3, \varphi\left(b_{3}\right)$ lies in $V_{6}$ and does not lie in $V_{4}+\left\langle c_{4}\right\rangle$, i.e., is of the form

$$
\varphi\left(b_{3}\right)=\lambda_{2} b_{2}+\lambda_{3} b_{3}+\lambda_{5} b_{5}+\lambda_{8} b_{8}+\mu_{2} c_{2}+\mu_{4} c_{4},
$$

where $\lambda_{3} \neq 0$. As $b_{3}$ is toral, $\varphi\left(b_{3}\right)$ is toral, and the equality $\varphi\left(b_{3}\right)=\varphi\left(b_{3}\right)^{[2]}$ is equivalent to the following quadratic system:

$$
\begin{aligned}
& \lambda_{2}=\lambda_{2} \lambda_{3} \\
& \lambda_{3}=\lambda_{3}^{2} \\
& \lambda_{5}=\lambda_{3} \lambda_{5} \\
& \lambda_{8}=\lambda_{3} \lambda_{8} \\
& \mu_{2}=\lambda_{3} \mu_{2} \\
& \mu_{4}=\lambda_{2}^{2} .
\end{aligned}
$$

Consequently,

$$
\varphi\left(b_{3}\right)=\lambda_{2} b_{2}+b_{3}+\lambda_{5} b_{5}+\lambda_{8} b_{8}+\mu_{2} c_{2}+\lambda_{2}^{2} c_{4}
$$

Assume $\lambda_{2} \neq 0$. Applying to both sides of the equality (11) the automorphism

$$
\exp \left(\mu_{2} c_{2}\right) \circ \Theta(\alpha) \circ \Phi\left(\lambda_{5}\right) \circ \Delta\left(\frac{1}{\sqrt{\lambda_{2}}}\right),
$$

where $\alpha$ satisfies the quadratic equation $\alpha^{2}+\alpha+\lambda_{8}=0$ (this is the only place were we need the assumption that any quadratic equation with coefficients in $K$ has a solution), we may assume

$$
\varphi\left(b_{3}\right)=b_{2}+b_{3}+c_{4} .
$$

The automorphism $\varphi$ maps the subalgebra $C_{\mathscr{L}}\left(b_{3}\right)$ to the subalgebra $C_{\mathscr{L}}\left(b_{2}+b_{3}+c_{4}\right)$. We have

$$
C_{\mathscr{L}}\left(b_{3}\right)=\left\langle b_{3}, b_{6}, b_{9}, c_{3}, c_{4}, c_{5}, d\right\rangle
$$

and

$$
C_{\mathscr{L}}\left(b_{2}+b_{3}+c_{4}\right)=\left\langle b_{2}+b_{3}+c_{4}, b_{5}+b_{6}, b_{8}+b_{9}, c_{2}+c_{3}, c_{4}, c_{5}, d\right\rangle .
$$

By Proposition 3, $\varphi\left(b_{6}\right)$ lies in $V_{6}^{\prime}$, thus does not contain terms with $b_{2}+b_{3}+c_{4}, b_{8}+b_{9}, c_{2}+c_{3}$, and $d$. Similarly, $\varphi\left(b_{9}\right)$ lies in $V_{6}^{\prime \prime}$, thus does not contain terms with $b_{2}+b_{3}+c_{4}, b_{5}+b_{6}, c_{2}+c_{3}$, and $d$. Therefore, we can write

$$
\begin{aligned}
& \varphi\left(b_{6}\right)=\alpha_{6}\left(b_{5}+b_{6}\right)+\alpha_{4} c_{4}+\alpha_{5} c_{5} \\
& \varphi\left(b_{9}\right)=\beta_{9}\left(b_{8}+b_{9}\right)+\beta_{4} c_{4}+\beta_{5} c_{5} \\
& \varphi(d)=\gamma_{3}\left(b_{2}+b_{3}+c_{4}\right)+\gamma_{6}\left(b_{5}+b_{6}\right)+\gamma_{9}\left(b_{8}+b_{9}\right)+\delta_{3}\left(c_{2}+c_{3}\right)+\delta_{4} c_{4}+\delta_{5} c_{5}+\gamma d
\end{aligned}
$$

for certain $\alpha_{i}, \beta_{i}, \gamma_{i}, \delta_{i}, \gamma \in K$. Then the equalities $\left[\varphi\left(b_{6}\right), \varphi(d)\right]=\varphi\left(b_{3}\right)$ and $\left[\varphi\left(b_{9}\right), \varphi(d)\right]=\varphi\left(b_{6}\right)$ are equivalent to

$$
\begin{gathered}
\alpha_{6} \gamma=1 \\
\alpha_{6} \delta_{3}+\alpha_{5} \gamma=1
\end{gathered}
$$

and

$$
\begin{gathered}
\beta_{9} \gamma=\alpha_{6} \\
\beta_{5} \gamma=\alpha_{4} \\
\beta_{9} \delta_{3}=\alpha_{5}
\end{gathered}
$$

respectively. It is easy to see that the system (12)-(16) is contradictory: for example, multiplying (16) by $\alpha_{6}$, and taking into account (13), we get $\beta_{9}+\alpha_{5} \beta_{9} \gamma=\alpha_{5} \alpha_{6}$, what, in its turn, together with (14) gives $\beta_{9}=0$, hence $\alpha_{5}=\alpha_{6}=0$, what contradicts (13).

Therefore $\lambda_{2}=0$. Applying to both sides of (11) the automorphism

$$
\Psi\left(\lambda_{5}\right) \circ \exp \left(\mu_{2} c_{2}\right) \circ \Theta\left(\sqrt{\lambda_{8}}\right),
$$


we may assume $\varphi\left(b_{3}\right)=b_{3}$. Consequently, the eigenspace $\left\langle b_{1}, b_{2}, b_{4}, b_{5}, b_{7}, b_{8}, c_{1}, c_{2}\right\rangle$ corresponding to the eigenvalue 1 of ad $b_{3}$, is invariant under $\varphi$, and we may write

$$
\varphi\left(b_{1}\right)=\lambda_{1} b_{1}+\lambda_{2} b_{2}+\lambda_{4} b_{4}+\lambda_{5} b_{5}+\lambda_{7} b_{7}+\lambda_{8} b_{8}+\mu_{1} c_{1}+\mu_{2} c_{2} .
$$

Applying to both sides of this equality the automorphism

$$
\exp \left(\lambda_{5} c_{5}\right) \circ \exp \left(\lambda_{8} c_{3}^{[2]}\right) \circ \exp \left(\lambda_{2} c_{4}\right) \circ \Phi\left(\lambda_{4}\right) \circ \Delta\left(\sqrt{\lambda_{1}}\right)
$$

we may assume $\lambda_{1}=1$ and $\lambda_{2}=\lambda_{4}=\lambda_{5}=\lambda_{8}=0$.

Then we have

$$
0=\varphi\left(b_{1}^{[4]}\right)=\varphi\left(b_{1}\right)^{[4]}=\mu_{1}^{2} b_{4}^{[2]}+\lambda_{7}^{4} b_{7}^{[2]}+\mu_{2}^{2} c_{3}^{[2]}+(\text { terms lying in } \mathscr{L}),
$$

what implies $\lambda_{7}=\mu_{1}=\mu_{2}=0$, and $\varphi\left(b_{1}\right)=b_{1}$. Further:

- $\varphi\left(c_{4}\right) \in\left\langle c_{4}\right\rangle$ by Proposition 3 ;

- $\varphi\left(b_{2}\right)=\left[\varphi\left(b_{1}\right), \varphi\left(c_{4}\right)\right] \in\left[b_{1},\left\langle c_{4}\right\rangle\right]=\left\langle b_{2}\right\rangle$;

- $\varphi\left(c_{2}\right) \in\left\langle c_{2}\right\rangle$ by Proposition 3 ,

- $\varphi\left(c_{3}\right)=\left[\varphi\left(b_{1}\right), \varphi\left(c_{2}\right)\right] \in\left[b_{1},\left\langle c_{2}\right\rangle\right]=\left\langle c_{3}\right\rangle ;$

- $\varphi\left(c_{1}\right)=\left[\varphi\left(b_{1}\right), \varphi\left(c_{3}\right)\right] \in\left[b_{1},\left\langle c_{3}\right\rangle\right]=\left\langle c_{1}\right\rangle$;

- $\varphi\left(c_{5}\right) \in\left\langle c_{5}\right\rangle$ by Proposition 3 ,

- $\varphi\left(b_{5}\right)=\left[\varphi\left(b_{1}\right), \varphi\left(c_{5}\right)\right] \in\left[b_{1},\left\langle c_{5}\right\rangle\right]=\left\langle b_{5}\right\rangle$;

- $\varphi\left(b_{6}\right)=\left[\varphi\left(b_{1}\right), \varphi\left(b_{5}\right)\right] \in\left[b_{1},\left\langle b_{5}\right\rangle\right]=\left\langle b_{6}\right\rangle$;

- $\varphi(d)=\left[\varphi\left(b_{1}\right), \varphi\left(c_{1}\right)\right] \in\left[b_{1},\left\langle c_{1}\right\rangle\right]=\langle d\rangle$;

- $\varphi\left(b_{7}\right)=\left[\varphi\left(c_{1}\right), \varphi(d)\right] \in\left[\left\langle c_{1}\right\rangle,\langle d\rangle\right]=\left\langle b_{7}\right\rangle$;

- $\varphi\left(b_{4}\right)=\left[\varphi\left(b_{7}\right), \varphi(d)\right] \in\left[\left\langle b_{7}\right\rangle,\langle d\rangle\right]=\left\langle b_{4}\right\rangle$;

- $\varphi\left(b_{8}\right)=\left[\varphi\left(c_{2}\right), \varphi(d)\right] \in\left[\left\langle c_{2}\right\rangle,\langle d\rangle\right]=\left\langle b_{8}\right\rangle$;

- $\varphi\left(b_{9}\right)=\left[\varphi\left(c_{3}\right), \varphi(d)\right] \in\left[\left\langle c_{3}\right\rangle,\langle d\rangle\right]=\left\langle b_{9}\right\rangle$.

Hence $\varphi$ is a diagonal automorphism, and by Lemma 4 we have $\varphi=\Delta(\lambda)$, where $\lambda^{-2}=1$. But then $\lambda=1$ and $\varphi=\operatorname{id} \mathscr{L}$.

\section{GRAdingS}

Having a supply of automorphisms of $\mathscr{L}$ at hand, and using the known correspondence between automorphisms and group gradings (formulated in full generality in the language of affine group schemes - see, for example, [EK, Proposition 1.36]), we may try to construct group gradings of $\mathscr{L}$.

In practice, this is achieved by extending the ground field $K$ to a suitable commutative ring $R$ (not necessary a field - we are dealing with the group scheme $R \rightarrow \operatorname{Aut}_{R}\left(\mathscr{L} \otimes_{K} R\right)$ ), extending an automorphism $\varphi$ of $\mathscr{L}$ to the automorphism $\bar{\varphi}$ of $\overline{\mathscr{L}}=\mathscr{L} \otimes_{K} R$ via $\bar{\varphi}(x \otimes r)=\varphi(x) \otimes r$, and considering eigenspaces $\overline{\mathscr{L}}_{\lambda}=\{x \in \overline{\mathscr{L}} \mid \bar{\varphi}(x)=\lambda x\}$. For a suitable choice of $\varphi$ and $R$, and a suitable homomorphism $\chi$ from the group generated by all eigenvalues $\lambda \in R$ to a group $G$, the eigenspaces will be "rational": $\bar{L}_{\lambda}=\mathscr{L}_{\chi(\lambda)} \otimes_{K} R$. The ensued grading $\mathscr{L}=\bigoplus_{\chi(\lambda)} \mathscr{L}_{\chi(\lambda)}$ will be a grading of $\mathscr{L}$ by $G$, even in the case when the eigenvalues $\lambda$ do not necessarily belong to the ground field.

For example, the diagonal automorphism $\Delta(\lambda)$ for the "generic" value of $\lambda$ (or, what is the same, for $\lambda \in K$ such that the order of $\lambda$ in the multiplicative group $K^{*}$ is $>7$ ) produces a $\mathbb{Z}$-grading

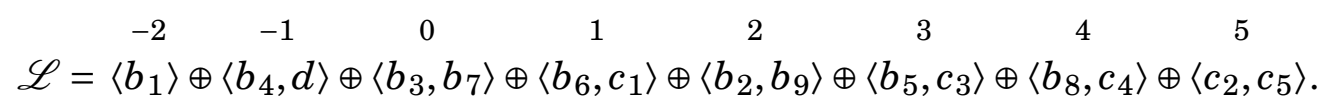

Specializing the automorphism $\Delta(\lambda)$ to the cases $\lambda^{n}=1, n \leq 7$, we obtain a $\mathbb{Z} / n \mathbb{Z}$-grading of $\mathscr{L}$. As the product of any two elements from the standard basis is either zero, or again an element from the standard basis, the decomposition of $\mathscr{L}$ into one-dimensional subspaces spanned by the basis element is a grading. This is a group grading, and its universal group ([EK, $\S 1.2])$ is isomorphic to the (additive) abelian group with generators $x, y$ (corresponding to elements $b_{1}, b_{4}$ 
respectively), and the relation $2 x=4 y$, what is easily seen to be isomorphic to the direct sum $\mathbb{Z} \oplus \mathbb{Z} / 2 \mathbb{Z}$. On the other hand, the thin decomposition exhibited in $\$ 3.4$ is a $(\mathbb{Z} / 2 \mathbb{Z})^{4}$-grading.

In general, it seems to be a difficult task to classify all group gradings of the Skryabin algebra. Even to determine whether the automorphisms of order 2 (exponential, $\Phi$, and $\Theta$ ) lead to $\mathbb{Z} / 2 \mathbb{Z}$ gradings seems to be far from trivial. Perhaps, it could be approached with the method of [KL].

\section{Comparison of the Skryabin Algebra With Algebras From the Eick List. A Bit MORE NUMEROLOGY}

In $[\mathrm{E}]$, a computer-generated list of simple Lie algebras over $\mathrm{GF}(2)$ of dimension $\leq 20$ is presented. The Skryabin algebra (defined over GF(2)) is not isomorphic to any of the 15-dimensional algebras in the list.

One way to see this, using data from $[\mathrm{E}]$, is to look at automorphisms, either at the group of exponential automorphisms $\operatorname{Exp}(\mathscr{L})$, or at the whole group $\operatorname{Aut}(\mathscr{L})$. As the ground field is $\mathrm{GF}(2)$, $\operatorname{Exp}(\mathscr{L})$ is isomorphic to the additive group $\mathrm{GF}(2) \oplus \mathrm{GF}(2) \oplus \mathrm{GF}(2) \oplus \mathrm{GF}(2)$, thus having order 16, what is different from all the 15-dimensional algebras in the list (including the new ones, number 7 and 8, dubbed by us here as $E i c k_{7}$ and $E i c k_{8}$ ) except for the non-alternating Hamiltonian algebra $P(2,1,1)$ (number 4 ). The order of $\operatorname{Aut}(\mathscr{L})$ is $2^{7}=128$ (over GF(2), there are no nontrivial diagonal automorphisms), what is, again, different from all the 15-dimensional algebras in the list.

Another way to distinguish between all these algebras, is to repeat for them the same pedestrian, but informative computations concerning tori and the sandwich subalgebra, as in $\$ 3.1$ and $\$ 2$. The following table accumulates some information about the 15-dimensional central simple algebras from the Eick list, whose derivation algebra is 19-dimensional (and coincides in all the cases with the 2-envelope). Here $T R$ denotes the absolute toral rank of the algebra, $N_{1}$ and $N_{m}$ denote, respectively, the number of toral elements, and of tori of the maximal dimension $T R$ in the 2-envelope, and $S$ is the dimension of the sandwich subalgebra.

\begin{tabular}{|l|r|c|c|r|}
\hline algebra & $T R$ & $N_{1}$ & $N_{m}$ & $S$ \\
\hline$W(4)$ & 2 & 256 & 1,536 & 10 \\
\hline$P(2,1,1)$ & 4 & 448 & 43,680 & 1 \\
\hline$P(3,1)$ & 3 & 384 & 10,752 & 5 \\
\hline$P(2,2)$ & 4 & 384 & 13,440 & 3 \\
\hline Eick $_{7}$ & 4 & 464 & 87,360 & 1 \\
\hline Eick $_{8}$ & 4 & 464 & 67,200 & 1 \\
\hline
\end{tabular}

Interestingly enough, all of these algebras which are of absolute toral rank 4 , i.e., $P(2,1,1)$, $P(2,2), E i c k_{7}$, and $E i c k_{8}$, also admit thin decompositions with respect to a lot (conjecturally with respect to all) of the 4-dimensional tori.

\section{OPEN QUESTIONS}

1. Is it true that two toral elements $h$ and $h^{\prime}$ in $\mathscr{L}$ are conjugate with respect to the automorphism group, if and only if $C_{\mathscr{L}}(h) \simeq C_{\mathscr{L}}\left(h^{\prime}\right)$ ?

2. Describe all gradings of the Skryabin algebra.

3. Conjecture. Any simple Lie algebra of dimension $>3$ over a field of characteristic 2 admitting a thin decomposition, has:

a) a proper simple graded subalgebra (with respect to this decomposition);

b) a graded subalgebra isomorphic either to $W$ or to $H$.

4. Classify simple finite-dimensional Lie algebras over an algebraically closed field of characteristic 2 , admitting a thin decomposition. 
5. Classify simple finite-dimensional $\mathbb{Z}$-graded Lie algebras over an algebraically closed field of characteristic 2 , such that all homogeneous components are of dimension $<3$. (Note that the Skryabin algebra belongs to this class, due to (17)).

\section{ACKNOWLEDGEMENTS}

Thanks are due to Bettina Eick, Dimitry Leites, and Alexander Premet for useful remarks and interesting discussions. Alexander Grishkov was supported by FAPESP, grant number 2018/23690-6 and CNPq, grant number 307824/2016-0; Marina Rasskazova during the work on this paper was visiting the University of São Paulo, and was supported by FAPESP, grant number 2018/11292-6.

\section{REFERENCES}

[BGLLS] S. Bouarroudj, P. Grozman, A. Lebedev, D. Leites, and I. Shchepochkina, Simple vectorial Lie algebras in characteristic 2 and their superizations, SIGMA 16 (2020), 089.

[E] B. Eick, Some new simple Lie algebras in characteristic 2, J. Symb. Comput. 45 (2010), no.9, 943-951.

[EK] A. Elduque and M. Kochetov, Gradings on Simple Lie Algebras, AMS, 2013.

[G] The GAP Group, GAP - Groups, Algorithms, and Programming, Version 4.11.0, 2020; https://www.gap-system.org/

[GG] A. Grishkov and M. Guerreiro, On simple Lie algebras of dimension seven over fields of characteristic 2, São Paulo J. Math. Sci. 4 (2010), no.1, 93-107.

[GGA] _ _ and W.F. de Araujo, On the classification of simple Lie algebras of dimension seven over fields of characteristic 2, São Paulo J. Math. Sci. 14 (2020), no.2, 703-713.

[GZ] _ and P. Zusmanovich, Deformations of current Lie algebras. I. Small algebras in characteristic 2, J. Algebra 473 (2017), 513-544.

[KL] A. Krutov and A. Lebedev, On gradings modulo 2 of simple Lie algebras in characteristic 2, SIGMA 14 (2018), 130.

[P] A.A. Premet, On Cartan subalgebras of Lie p-algebras, Izv. Akad. Nauk SSSR Ser. Mat. 50 (1986), no.4, 788-800 (in Russian); Math. USSR Izvestija 29 (1987), no.1, 145-157 (English translation).

[Sk] S. Skryabin, Toral rank one simple Lie algebras of low characteristics, J. Algebra 200 (1998), no.2, 650-700.

[St] H. Strade, Simple Lie Algebras over Fields of Positive Characteristic. Vol I. Structure Theory, 2nd ed.; Vol. II. Classifying the Absolute Toral Rank Two Case, 2nd ed.; Vol. III. Completion of the Classification, De Gruyter, 2017, 2017, 2012.

[V-L1] M. Vaughan-Lee, The Restricted Burnside Problem, 2nd ed., Oxford Univ. Press, 1993.

[V-L2] _ Simple Lie algebras of low dimension over GF(2), LMS J. Comput. Math. 9 (2006), 174-192.

[W] B. Weisfeiler, On the structure of the minimal ideal of some graded Lie algebras of characteristic $p>0$, J. Algebra 53 (1978), no.2, 344-361.

(Alexander Grishkov) University of SÃo PaUlo, Brazil and Omsk F.M. Dostoevsky State University, RUSSIA

Email address: shuragri@gmail.com

(Henrique Guzzo Jr.) University of São PaUlo, Brazil

Email address: guzzo@ime.usp.br

(Marina Rasskazova) OMsK State Technical University, Russia

Email address: marinarasskazova@yandex.ru

(Pasha Zusmanovich) University of Ostrava, Czech RePUbliC

Email address: pasha.zusmanovich@gmail.com 